\title{
Spiral Growth of Fungus Colonies
}

\author{
By M. F. MADELIN, D. K. TOOMER AND JILL RYAN \\ Department of Botany, The University, Bristol BS8 $1 U G$
}

(Received 25 November 1977)

\begin{abstract}
Sordaria fimicola on $0.02 \%$ malt extract agar displayed spiralling of the colony in a clockwise direction (viewed from above the agar plate). Only hyphae at the agar surface contributed to this spiral aspect. The direction of curvature of individual hyphae was related to their supporting surface; hyphae curved to the right as they advanced over the surface. This sustained departure from straight growth is attributed to a displacement of the plastic apex of the hypha to the right as a result of its own clockwise axial rotation (as viewed tipwards from within the hypha). Attempts to observe directly the rotation of the apex failed. Calculations show that the rate of angular rotation of an individual leading hypha was of the order of $500^{\circ}$ per millimetre of extension, a rate similar to that in several other cells which spiral as they grow. However the angle of spiral growth was very small (about $0.7^{\circ}$ ). Out of 157 isolates of fungi tested, 21 showed pronounced spiralling of their colonies on $0.02 \%$ malt extract agar and 39 showed weak spiralling, whence it is inferred that spiral growth of individual vegetative hyphae is of widespread occurrence amongst fungi.
\end{abstract}

\section{INTRODUCTION}

Spiral patterns of growth are sometimes encountered in the colonies of filamentous fungi. Such patterns arise from various causes. For example an endogenous rhythm of sporulation may produce a colony which forms an Archimedes spiral that grows usually by one revolution for each cycle of the endogenous rhythm (Bourret, Lincoln \& Carpenter, $1969,1971)$. However, spiral growth occurs most commonly when, as Ritchie (1960) reports, 'instead of growing radially from a point of inoculation, as fungus mycelium ordinarily does, some hyphae grow out with a definite bend'. There have been few studies of this particular phenomenon apart from that of Ritchie who examined the counter-clockwise spiralling (as viewed from above the culture plate) of Aspergillus ustus, Phoma sp. and two Curvularia species. He found that the manifestation of spiralling depended on the incubation temperature, $\mathrm{pH}$ and composition of the culture medium; that it never appeared in colonies on liquid media; that the spiralling was not governed by electrical or gravitational fields in the environment; and that there was no consistent correlation of spiralling and geographic location at different sites in the North American continent. He was unable to advance an explanation of the phenomenon.

Having discovered a cultural condition under which spiral growth is manifested by Sordaria fimicola (Roberge) Cesati \& de Notaris, we have studied the phenomenon and offer an explanation. 


\section{METHODS}

All the species of fungi used were from the culture collection of the Department of Botany of the University of Bristol. Strains are identified by their numbers in that collection.

Malt extract agars (MA) of different concentrations were prepared from commercial malt extract (Boots the Chemists, Ltd) and contained $2 \%(\mathrm{w} / \mathrm{v})$ agar. Media were made up in distilled water. Agar plates were prepared in $85 \mathrm{~mm}$ diam. plastic disposable Petri dishes filled to a depth of 2 to $3 \mathrm{~mm}$. Cultures were incubated in a $22{ }^{\circ} \mathrm{C}$ constant temperature room or in $25^{\circ} \mathrm{C}$ incubators. Most experiments were performed first at $22{ }^{\circ} \mathrm{C}$ and repeated at $25^{\circ} \mathrm{C}$ with similar results. For observations on spiral growth of colonies, up to eight inocula were distributed on each plate. Each inoculum consisted of an approximately $2 \mathrm{~mm}$ diam. plug of agar cut from the margin of a colony grown on the same medium. Observations were made after about $20 \mathrm{~h}$ and thereafter at appropriate intervals.

Photographs were taken on Ilford FP 4 film by means of a Zeiss photomicroscope. All references to clockwise and counter-clockwise spiralling of colonies refer to the appearance when viewed by eye from above the culture surface. The optical inversion in photomicrographs means that clockwise spiralling appears in them as counter-clockwise, and vice versa. All references to clockwise and counter-clockwise rotation of individual hyphal tips about their axes refer to the direction when viewed acropetally, i.e. viewed tipwards from within the hyphae.

\section{RESULTS AND DISCUSSION}

\section{Circumscription of the spiral growth phenomenon under study}

Only one sort of spiral colonial growth is considered in the present study. It is that which involves the hyphae at the surface of weak culture media which support only thin mycelia. It was observed when Sordaria fimicola (A31) was grown on plates of $0.02 \%$ (w/v) MA. There was almost total absence of aerial mycelium, while those hyphae at the surface spiralled in a clockwise direction (Fig. 1). Hyphae immersed in the agar showed no consistent curvatures. First observed in S. fimicola, such spiral growth has now been seen in a number of species (see below).

\section{Spiral growth of surface hyphae of Sordaria fimicola}

This was best displayed on $0.02 \%$ MA. It was less evident on $0.1 \%$ MA and not apparent on $2 \%$ MA except occasionally in very young colonies ( $<24 \mathrm{~h}$ old). On $0.02 \%$ MA the colonies were very thin. To investigate whether the isolate was consistent in its spiralling, seven clones were derived from single ascospores of the parent strain and grown on $0.02 \%$ MA at $22^{\circ} \mathrm{C}$. All seven spiralled clockwise. To test whether an external influence capable of orienting the hyphae was responsible for the spiralling, cultures were incubated upside down, but they still spiralled clockwise (as viewed from above the culture surface).

If the direction of curvature of hyphae to the left or right was internally determined, it should have been possible to cause a reversal of curvature by excising a clockwise-spiralling colony and placing it face down on the surface of a fresh plate of $0.02 \%$ MA. In fact, the now sandwiched surface hyphae grew more or less straight and radially till they emerged from the sandwich when they resumed curvature in a clockwise direction with respect to the new surface on which they grew. The direction of curvature was thus related to the supporting surface.

This was confirmed by the following experiment. A rectangular coverglass was coated on one face with a thin film of $0.02 \% \mathrm{MA}$ and then placed like an ascending ramp, propped on a small cube of agar, in the path of the advancing clockwise-spiralling margin of a colony. When the colony front passed beneath the edge of the coverglass, some hyphae continued to advance on the surface of the agar plate while some advanced on the coated under-surface of the coverglass. Viewed from above the plate (Fig. 2), the colony continued to spiral clockwise on the surface of the agar plate but counter-clockwise on the coverglass. The direction of spiralling was thus expressly related to the surface to which the hyphae were attached and not to any influences external to the culture vessel. On a suitable surface, a superficial hypha of Sordaria fimicola curved towards the right as it advanced whatever the orientation of the surface. 


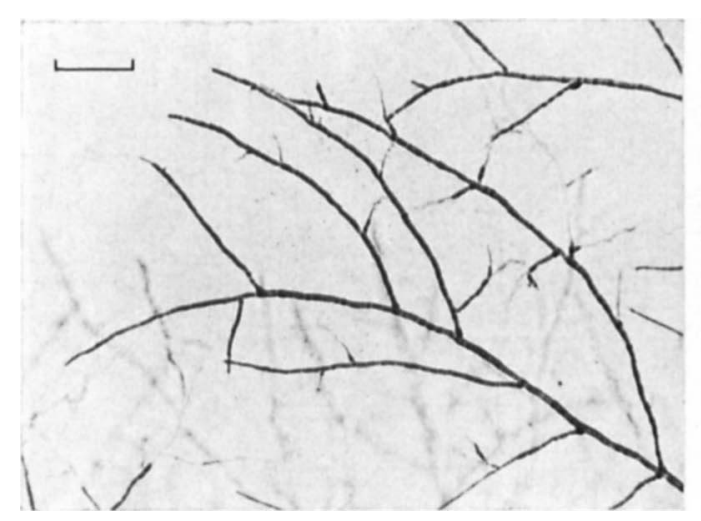

Fig. 1

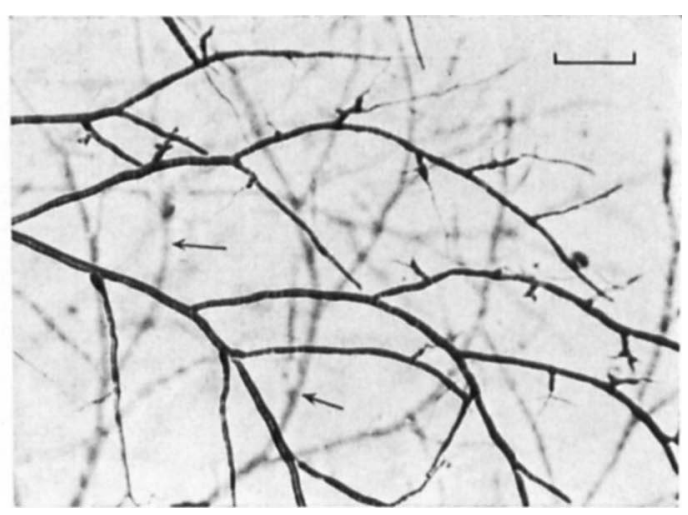

Fig. 2

Fig. 1. Clockwise-spiralling colony (optically inverted in photograph) of Sordaria fimicola on $0.02 \%$ malt extract at $22^{\circ} \mathrm{C}$. Bar marker represents $100 \mu \mathrm{m}$.

Fig. 2. Hyphae of Sordaria fimicola at $22{ }^{\circ} \mathrm{C}$ curving as they advance across the under-surface of a coverglass propped on the surface of $0.02 \%$ malt extract agar, over which other hyphae (unfocused, arrows) are curving in the opposite direction. As they advance, both groups of hyphae curve to the right in relation to their support. (N.B. Optically inverted.) Bar marker represents $100 \mu \mathrm{m}$.

\section{Possible mechanism of spiral growth of surface hyphae of Sordaria fimicola}

If the curvature of a given hypha were the result of an internally determined sustained difference in the growth rates of its two opposite sides, it is difficult to see why the fastergrowing side of the hypha should always be located on the left side whatever the inoculum and its orientation. For example, the probability that the germ tubes from seven ascospores happened by chance to lie upon the agar surface with their faster-growing sides in such positions that their tendencies for curvature led all of them to grow in a right-handed (i.e. clockwise) spiral is only $0.8 \%$. Because the right-handedness of curvature is, as shown above, dependent on the relationship of the hyphae to a supporting surface it is in this relationship that any hypothesis to explain the curvature must be rooted.

We advance the following hypothesis. We first postulate that the apex of each vegetative hypha rotates about its axis in a clockwise direction with respect to the subapical regions of the hypha as it grows. Because the subapical regions of the hypha cannot rotate owing to their anchorage to the substratum, the tip itself must rotate clockwise as it advances. The clockwise rotation of the delicate and somewhat plastic tip as it advances displaces it to the right by a lateral rolling action over its supporting surface (Fig. 3). The sustained slight lateral displacement imparts a curvature towards the right to the hypha which is formed.

This hypothesis is consistent with the observations on Sordaria fimicola reported above. The absence of curvature on nutritive media (e.g. 2 or $3 \% \mathrm{MA}$ ) appears to be the result of the much greater density of the colony and the consequently greater degree of interference between adjacent hyphae. When the curvature of surface hyphae brings them into proximity with others they often then curve differently. Curvature is manifested only on very dilute media probably because under these conditions chemotropic responses, which otherwise override and obscure the intrinsic tendency to curvature, are reduced.

We thus suggest that the primary biological phenomenon involved is the axial rotation of the hyphal apex, and that spiral colonial growth is a secondary manifestation. Attempts made to detect this rotation were unsuccessful. We attempted to deposit markers (which included particles of ink and powdered charcoal, conidia of Cunninghamella elegans and of species of Penicillium, and cells of Escherichia coli) on the apices of vegetative hyphae growing in humid air and in liquid culture medium, but were not able to mark the small and fragile apex for long enough to establish whether it rotated. The reasonableness of the 


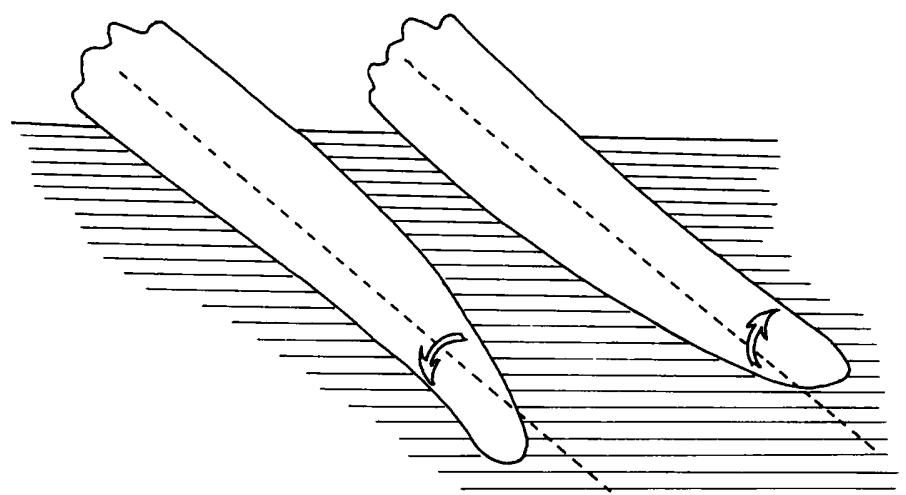

Fig. 3. Hypothetical lateral displacement of plastic extension zones of hyphae to the left or right with respect to the substratum according to whether the apices rotate counter-clockwise or clockwise, respectively.

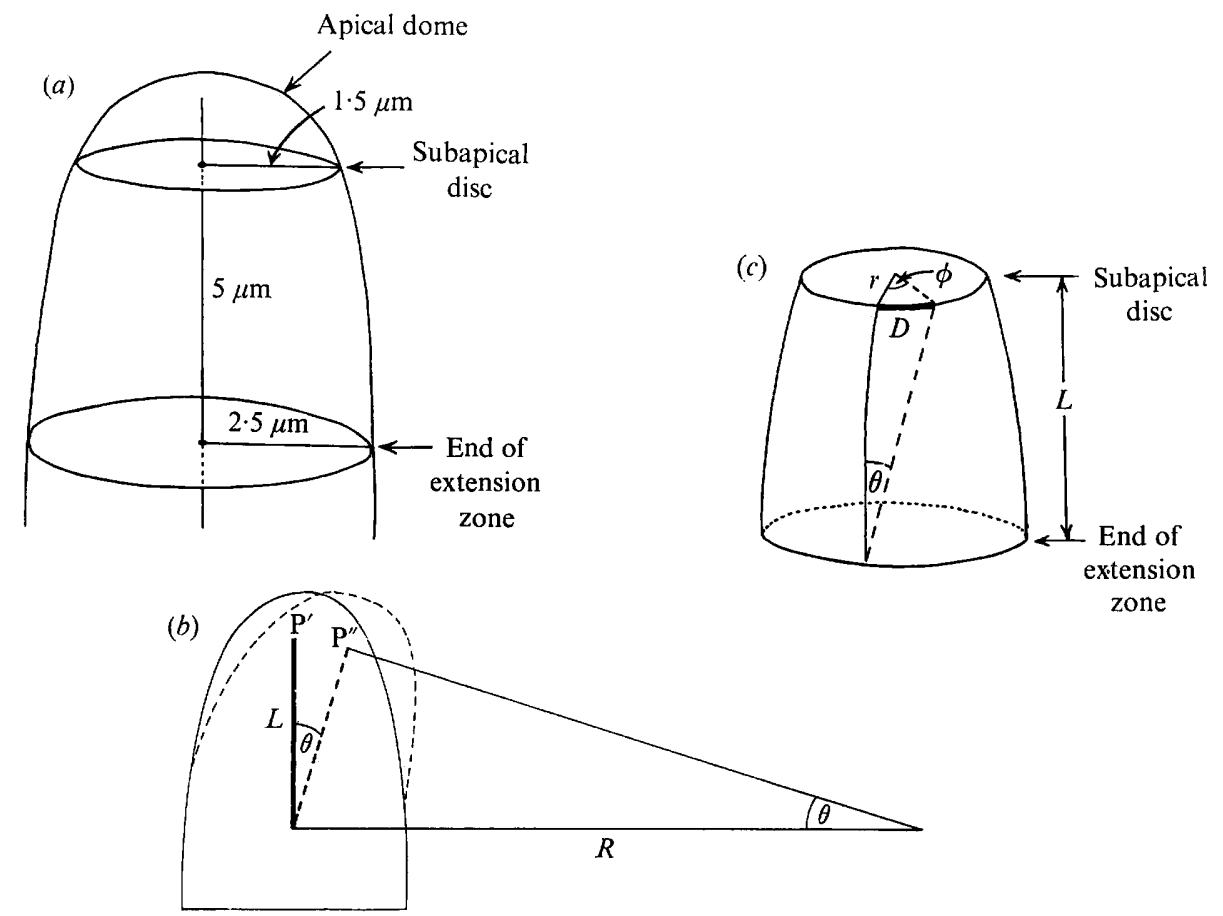

Fig. 4. (a) Generalized model of apex of superficial vegetative hypha of Sordaria fimicola. $(b)$ Derivation of lateral displacement (as distance $\mathbf{P}^{\prime} \mathbf{P}^{\prime \prime}$, and as angle $\theta$ ) of apex of hypha from knowledge of effective length of the extension zone $(L)$ and radius of curvature $(R)$ of path of the advancing hypha (see text). (c) Derivation of rate of angular rotation $(\Delta \phi / \Delta L)$ of 'subapical disc' (see text).

postulate is nevertheless supported by the well known example of the sporangiophore of Phycomyces blakesleeanus (Oort, 1931; Castle, 1942; Preston, 1948; Middlebrook \& Preston, 1952a,b). Explanations for the rotational growth (so-called 'spiral growth') of the sporangiophore of Phycomyces have invoked as a basic cause the structural properties of the hyphal wall (Oort, 1931; Castle, 1942; Preston, 1948; Roelofsen, 1950; Middlebrook \& Preston, $1952 a, b)$, and similar correlations have been made in other cells, for example, of algae (Frei \& Preston, 1961).

In order to compare the postulated rotation of the hyphal tips of Sordaria fimicola with 
the rotation directly observed in certain other systems, a quantitative description of the tip is necessary. The apices of vegetative hyphae at the surface of dilute malt extract agar are, like the hyphae themselves, variable in size but certain generalizations can be made. The mature width of hyphae is usually between 3.5 and $7 \mu \mathrm{m}$, and is attained quickly, usually within $6 \mu \mathrm{m}$ of the tip. The extremity of the tapered tip is approximately hemispherical with a radius of curvature about two-thirds that of mature regions of the hypha. The length of the subtending extension zone may be inferred from the distance from the apex at which a hypha attains maximal width (Trinci \& Halford, 1975); in Sordaria fimicola this is commonly $6 \mu \mathrm{m}$. By observing minor irregularities and branch initials on the sides of the subapical regions of growing hyphae and noting that they did not follow the advancing apex. it was independently concluded that the extension zone, and therefore the zone within which any rotation of the apex was generated, could not have been more than $7.5 \mu \mathrm{m}$ in length. The radii of sustained growth curvatures of surface hyphae were variable, but commonly were between 200 and $800 \mu \mathrm{m}$, and mostly about $400 \mu \mathrm{m}$.

These data may be used to construct a model of a generalized apex of a superficial hypha of Sordaria fimicola so that certain conclusions about the order of magnitude of the rotational growth may be derived. The generalized model is shown in Fig. 4(a). It is assumed that although the angular rotation of the extreme apex may be greater than that of regions a short distance back, the apical dome itself probably contributes nothing to the lateral displacement of the tip because, firstly, it is not in contact with the solid substratum and, secondly, even if it were, its rapidly diminishing cross-sectional circumference would more than offset the advantage of increasing rotational velocity. It has therefore been assumed for the purposes of constructing a model that in a hypha of mature diameter of $5 \mu \mathrm{m}$ it is the circumference of a hypothetical subapical disc where the hypha is $3 \mu \mathrm{m}$ wide, about 1 to $1.5 \mu \mathrm{m}$ behind the extreme apex, which contributes the greatest lateral displacement. Between it and the proximal end of the extension zone where there is no rotation there would be a progressively diminishing contribution.

From the observed curvature of the hypha as it advances (Fig. $4 b ; L=5 \mu \mathrm{m}, R=400$ $\mu \mathrm{m})$, the right-hand displacement of the apex can be calculated in terms of both angle $(\theta)$ and distance $\left(\mathrm{P}^{\prime} \mathrm{P}^{\prime \prime}\right)$.

$$
\begin{gathered}
\sin \theta=L / R=5 / 400=0.013 \quad \text { whence } \theta=0.7^{\circ} \\
\mathrm{P}^{\prime} \mathrm{P}^{\prime \prime}=2 L \sin (\theta / 2)=0.06 \mu \mathrm{m}
\end{gathered}
$$

The angular rotation of the subapical disc whose circumference generates the calculated lateral displacement can be expressed in relation to the extension of the hypha (cf. Frei \& Preston, 1961) and can be calculated thus (Fig. 4c):

$$
\text { Rate of angular rotation }=\frac{\Delta \phi}{\Delta L}=\frac{D}{2 \pi r} \cdot \frac{360}{L} \quad \text { (in }{ }^{\circ} \mathrm{mm}^{-1} \text { ) }
$$

where $D$ is the circumferential displacement of the margin of the 'subapical disc' in growing through the distance $L$ (in $\mathrm{mm}$ ), and is quantitatively equal to $\mathrm{P}^{\prime} \mathrm{P}^{\prime \prime}$, i.e.

Rate of angular rotation $=458^{\circ} \mathrm{mm}^{-1}$ for the above values.

A rotation rate of about $500^{\circ} \mathrm{mm}^{-1}$ is equivalent to a rotation of only $2 \cdot 5^{\circ}$ while the apex lengthens by $5 \mu \mathrm{m}$. The smallness of this rotation probably explains our failure to detect axial rotation by direct observation.

The data of Castle (1942) for a Stage 4B sporangiophore of Phycomyces indicate a rate of angular rotation of $300^{\circ} \mathrm{mm}^{-1}$. It is clearly of the same order as the rate invoked here to explain spiral colonial growth. Frei \& Preston (1961), listing rates of rotation for algae, report $350^{\circ} \mathrm{mm}^{-1}$ for Chaetomorpha melagonium, $1050^{\circ} \mathrm{mm}^{-1}$ for Cladophora rupestris and $200^{\circ} \mathrm{mm}^{-1}$ for Cladophora prolifera. Again, the orders of magnitude are similar. However when one compares the angles of spiral growth, that estimated for Sordaria fimicola $\left(0.7^{\circ}\right)$ is minute compared with estimates for Phycomyces, e.g. 13.9 ${ }^{\circ}$ (range 3.8 to 
Table 1. The occurrence of spiral colonial growth in filamentous fungi grown on $0.02 \%(w / v)$ malt extract agar

A. Pronounced clockwise spiralling Iodophanus carneus (A118) Pestalotiopsis guepini (R87) Sordaria fimicola (A31)
Sordaria fimicola (A102)

Sordaria fimicola (A113)

Sordaria fimicola (albino) (A101)

Ulocladium sp. (albino) (R84)

Wardomyces pulvinata (R55)

Rhizoctonia solani (R18)

Rhizopus arrhizus (Z33)

Stemphylium ilicis (R58)

Thielaviopsis basicola (R62)

Pleospora infectoria (A110)

Polyporus brumalis (B13)

Ryparobius sp. (A94)

Saprolegnia sp. (O11)

Phycomyces blakesleeanus (-) (Z50b)

Pirella sp. $(+)(\mathrm{Z} 57)$

Pirella sp. (-) (Z58)

Podospora anserina (A99)

Pythium debaryanum (O1)

Rhizopus stolonifer (Z14a)

Syncephalastrum racemosum (Z72)

Ulocladium $\mathrm{sp}$. (R92)

Penicillium brevicompactum (A82)

Penicillium clavigerum (A65)

Phycomyces blakesleeanus $(+)(\mathrm{Z} 50 \mathrm{a})$

Heterobasidion annosum (B17)

Hyalostachybotrys bisbyi (R78)

Leptographium sp. (R69)

Memnoniella echinata (R66)

Metarrhizium anisopliae (R38)

Monascus ruber (A8)

Monascus ruber (A106)

Monotospora sp. (R67)

Mortierella ramanniana (Z59)

Mycotypha africana (Z60)

Myxotrichum setosum (A93)

Nectria cinnabarina (A100)

Nectria galligena (A36)

Oidiodendron $\mathrm{sp}$. (R68)

Paecilomyces farinosus (R47)

Paecilomyces farinosus (R48)

Penicillium albicans (A73)

Penicillium camemberti (A57)

Penicillium cyclopium (A80)

Penicillium claviforme (A17)

Penicillium egyptiacum (A59)

Penicillium expansum (A62)

Penicillium frequentans (A72)

Penicillium janthinellum (A52)

Penicillium luteum (A81)

Penicillium roqueforti (A56)

Penicillium sp. (A97)

Pestalotia sp. (R96)

Pholiota adiposa (B5)

Pholiota adiposa (B22)

Phycomyces blakesleeanus ( + ) (Z54)

Phycomyces blakesleeanus ( +) (Z11b)
Phycomyces blakesleeanus (-) (Z55)

Phytophthora cactorum (O6)

Pirella circinans $(+)(\mathrm{Z22a})$

Pleurotus ostreatus (B25)

Podospora anserina (A96)

Podospora anserina (A98)

Podospora curvicolla (A95)

Podospora curvula (A112)

Podospora setosa (A115)

Polyporus betulinus (B16)

Polystictus versicolor (B8)

Pyronema confluens (A38)

Pythium middletonii (O14)

Rhizopus arrhizus (Z34)

Schizophyllum commune (B15)

Sclerotinia fructigena (R2)

Scopulariopsis brevicaulis (R71)

Sordaria inaequalis (A116)

Stachybotrys atra (R65)

Stachybotrys atra var. cylindrospora (R80)

Stachybotrys dichroa (R81)

Stilbella sp. (R63)

Syncephalastrum racemosum (Z56)

Thamnidium elegans (Z48)

Trichoderma viride (R4)

Trichothecium roseum (R12)

Trichothecium roseum ( $\mathrm{R} 97$ )

Tritirachium sp. (R98)

Ulocladium sp. (R83)

Zygorhynchus macrocarous (Z4)

Zygorhynchus moelleri (Z12)

Zygorhynchus sp. (Z49) 
$27.8^{\circ}$ ) (Middlebrook \& Preston, $1952 b$ ), $12.9^{\circ}$ (range 2.6 to $26.5^{\circ}$ ) (Castle, 1936), and $5.8^{\circ}$ (range 1.8 to $13.5^{\circ}$ ) (Oort, 1931). This may be a consequence of the difference in size of hyphae of Sordaria and sporangiophores of Phycomyces, for the latter are about 10 times wider. For the same rate of angular rotation $\left(458^{\circ} \mathrm{mm}^{-1}\right)$ but with a 10 -fold wider hypha, the angle of spiral growth in Sordaria would be that whose tangent is 10 -fold greater (since $\tan \theta=(\phi / 360) 2 \pi r / L$; Fig. $4 c)$, namely $7^{\circ}$, a figure within the range of values determined for Phycomyces.

Several workers (e.g. Oort, 1931 ; Castle, 1942; Preston, 1948; Roelofsen, 1950; Middlebrook \& Preston, 1952a; Frei \& Preston, 1961) have suggested that spiral growth of cells may be a consequence of the orientation of submicroscopic physical structures (micelles, microfibrils) within their walls. However, as Oort (1931) remarked, the directions of spiral growth and structure theoretically need not coincide. Middlebrook \& Preston (1952a) emphasize that the spiral of growth corresponds to an orientation of hypothetical fibrils in the wall whose properties depend on the dispersion of real microfibrils about this spiral direction. Consequently the inference that there is only a very small angle of spiral growth in Sordaria fimicola hyphae does not allow one to predict the orientation of microfibrils within their walls.

\section{Survey of the occurrence of spiral colonial growth in filamentous fungi}

The possibility that spiral colonial growth can be manifested by fungi other than the isolate studied above was investigated. A total of 157 isolates of different fungi were inoculated on to $0.02 \%$ MA and the growth of their colonies was examined. The results are presented in Table 1. 'Pronounced spiralling' means that most leading hyphae and first-order branches were clearly curved consistently one way or the other. 'Weak spiralling' means either that curvatures were slight or that they were clearly manifested by only some of the leading hyphae.

The results show that 21 isolates $(13.4 \%$ ) distributed among 17 species showed pronounced spiralling of their colonies, $8(5 \cdot 1 \%)$ being clockwise and $13(8 \cdot 3 \%)$ counter-clockwise. Another 39 isolates $(24.8 \%)$ showed weak curvatures, again with more counter-clockwise $(26 ; 16.6 \%)$ than clockwise $(13 ; 8.3 \%)$. The majority of isolates $(97 ; 61.8 \%)$ showed no curvatures or only inconsistent ones. Spiralling, either pronounced or weak, was seen in representatives of all five subdivisions of the fungi. There was thus no evidence that the occurrence and sort of spiralling served to differentiate the major taxa.

There was evidence of strain variation in some species. Thus Botrytis cinerea R1b and Rhizopus arrhizus Z33 showed spiralling whereas B. cinerea R1a and R. arrhizus Z34 did not.

\section{Conclusion}

The sort of spiral colonial growth considered here was seen only under rather closely circumscribed conditions since chemotropic responses in dense colonies appeared to obscure it, and departure of a hypha from the agar surface into the air or the substrate below eliminated the physical circumstance (unilateral support) necessary for curvature to develop. Consequently this phenomenon probably contributes little to the usual architecture of fungus colonies. Its significance lies chiefly in its implication that spiral growth of individual fungal hyphae is of common occurrence, a conclusion which fulfils Castle's (1942) prophetic remark that 'it is likely that suitable growth studies will reveal that the incidence of spiral growth is more general than has been supposed'. In view of preliminary results which indicate that culture conditions (including incubation temperature and atmospheric carbon dioxide concentration) influence the manifestation and direction of hyphal curvature of certain fungi, it is possible that some species which did not appear to spiral (Table 1) might do so under other conditions. Although it is not evident that colony spiralling differentiates or characterizes the major taxa, it might nevertheless serve as an additional taxonomic criterion for lesser taxa. 
We thank Dr A. R. Paterson, School of Mathematics, University of Bristol, for commenting helpfully on the mathematical aspects of this paper.

\section{REFERENCES}

Bourret, J. A., Lincoln, R. G. \& CARPenter, B. H. (1969). Fungal endogenous rhythms expressed by spiral figures. Science 166, 763-764.

BOURRET, J. A., LinCOLN, R. G. \& CARPENTER, B. H. (1971). Modifications of the period of a noncircadian rhythm in Nectria cinnabarina. Plant Physiology 47, 682-684.

CASTLE, E. S. (1936). The origin of spiral growth in Phycomyces. Journal of Cellular and Comparative Physiology 8, 493-502.

CASTle, E. S. (1942). Spiral growth and reversal of spiralling in Phycomyces, and their bearing on primary wall structure. American Journal of Botany 29, 664-672.

Frei, E. \& Preston, R. D. (1961). Cell wall organization and wall growth in the filamentous green algae Cladophora and Chaetomorpha. II. Spiral structure and spiral growth. Proceedings of the Royal Society B155, 55-77.

Middlebrook, M. J. \& Preston, R. D. (1952a). Spiral growth and spiral structure. III. Wall structure in the growth zone of Phycomyces. Biochimica et biophysica acta 9, 32-48.
Middlebrook, M. J. \& Preston, R. D. (1952b). Spiral growth and spiral structure. IV. Growth studies and mechanical constants in the cell wall. Biochimica et biophysica acta 9, 115-126.

OorT, A. J. P. (1931). The spiral growth of Phycomyces. Proceedings. Koninklijke nederlandse akademie van wetenschappen 34, 564-575.

Preston, R. D. (1948). Spiral growth and spiral structure. I. Spiral growth in sporangiophores of Phycomyces. Biochimica et biophysica acta 2, 155-166.

Ritchie, D. (1960). Spiral growth of fungus colonies. Growth 24, 391-400.

Roelofsen, P. A. (1950). The origins of spiral growth in Phycomyces sporangiophores. Acta botanica neerlandica 42, 72-110.

Trinci, A. P. J. \& Halford, E. A. (1975). The extension zone of stage I sporangiophores of Phycomyces blakesleeanus. New Phytologist 74, 81-83. 